\title{
Einsteinian Gravitational Field of a Heterogeneous Fluid Sphere.
}

By Mr. Jyotirmaya Ghosh, M.A., Dacca University.

(Received 24th May 1926. Read 5th June 1926.)

\section{$\S 1$. Introductory.}

The field-equations of gravitation in Einstein's theory have been solved in the case of an empty space, giving rise to de Sitter's spherical world. ${ }^{1}$ In the case of homogeneous matter filling all space, the solution gives Einstein's cylindrical world. ${ }^{2}$ The field corresponding to an isolated particle has been obtained by Schwarzchild. ${ }^{3}$ He has also obtained a solution for a fluid sphere with uniform density, ${ }^{4}$ a problem treated also by Nordström ${ }^{5}$ and de Donder. ${ }^{\circ}$ A new solution of the gravitational equations has been obtained in this paper, which corresponds to the field of a heterogeneous fluid sphere, the density at any point being a certain function of the distance of the point from the centre. The law of density is quite simple and such as to give finite density at the centre and gradually diminishing values as the distance from the centre increases, as might be expected of a natural sphere of fluid of large radius. The general problem of the fluid sphere with any arbitrary law of density cannot be solved in exact terms. It will be seen, however, from a theorem obtained in this paper, that the solution depends on a linear differential equation of the second order with variable coefficients involving the density, and thus the

${ }^{1}$ EdDinoton, Mathematical Theory of Relativity, Art. 45.

${ }^{2}$ Ibid, Art. 67.

' Ibid, Art. 38. Über das Gravitationsfeld eines Massenpunktes nach der Einsteinschen Theorie, Berlin-Sitzungsberichte, 1916, p. 189.

4 Über das Gravitationafeld einer Kugel aus incompressibler Flüssigkeit, Berlin Sitz., 1916, p. 426.

'Calculation of some special cases in Einstein's Theory of Aravilation, Proc. Amsterdam Acad., 21 (1919).

${ }^{8}$ La Gravifique Einsteinniene. 
laws of density for which the problem admits of exact solution are those for which the above coefficients satisfy the conditions of integrability of the differential equation. An approximate solution for any law of density may be obtained by the method of series.

\section{\$2. The Equations.}

On account of spherical symmetry, we assume the space-time to be the Riemannian space whose metric is

$$
d s^{2}=-e^{\lambda} d r^{2}-r^{2} d \theta^{2}-r^{2} \sin ^{2} \theta d \phi^{2}+\epsilon^{\nu} d t^{2},
$$

where the velocity of light is taken as unity, and $\lambda$ and $v$ are functions of $r$ only.

The field-equations of gravitation are

$$
K_{p_{t}}-\frac{1}{2} g_{p_{t}} K=-8 \pi T_{p_{t}},(p, q=1,2,3,4)
$$

where $K_{p q}=$ Contracted Riemann-Christoffel tensor,

$$
\begin{aligned}
& K=g_{p q} K^{p q}=\text { constant curvature, } \\
& T_{p q}=\text { Material-energy-tensor. }
\end{aligned}
$$

Now, we have

$$
\begin{aligned}
& g_{11}=-e^{\lambda}, g_{22}=-r^{2}, g_{33}=-r^{2} \sin ^{2} \theta, g_{14}=e^{y} \\
& g^{11}=-e^{-\lambda}, g^{22}=-r^{-2}, g^{33}=-(r \sin \theta)^{-2}, g^{44}=e^{-\nu}
\end{aligned}
$$

Hence, $g_{p q}(p \neq q)=0, K_{p q}^{r}(p \neq q)=0$, and the equations (2) reduce to the four equations (with no summation convention)

$$
K_{p p}-\frac{1}{2} g_{p p} K=-8 \pi T_{p p},(p=1,2,3,4)
$$

Since $T_{11}^{\prime}=g_{\sigma_{1}} T_{1}^{0}=g_{11} T_{1}^{1}=-e^{\lambda} T_{1}^{1}$,

$$
T_{20}^{\prime}=-r^{2} T_{2}^{2}, T_{33}=-r^{2} \sin ^{2} \theta T_{3}^{3}, T_{44}=\epsilon^{\nu} T_{4}^{4},
$$

and

$$
\begin{aligned}
& K_{11}-\frac{1}{2} g_{11} K=-\frac{1}{r} \frac{d v}{d r}-\frac{1-e^{\lambda}}{r^{2}}, \\
& K_{02}-\frac{1}{2} g_{22} K=-r^{2} e^{-\lambda} \\
& \quad\left[\frac{1}{2} \frac{d^{2} v}{d r^{2}}-\frac{1}{4} \frac{d \lambda}{d r} \frac{d \nu}{d r}+\frac{1}{4}\left(\frac{d v}{d r}\right)^{2}+\frac{1}{2 r}\left(\frac{d \nu}{d r}-\frac{d \lambda}{d r}\right)\right], \\
& K_{33}-\frac{1}{2} g_{33} K=\sin ^{2} \theta\left(K_{22}-\frac{1}{2} g_{22} K\right), \\
& K_{44}-\frac{1}{2} g_{44} K=e^{p-\lambda}\left(-\frac{1}{r} \frac{d \lambda}{d r}+\frac{1-e^{\lambda}}{r^{2}}\right),
\end{aligned}
$$


the equations (4) are

$$
\begin{aligned}
& e^{-\lambda}\left(\frac{1}{r} \frac{d \nu}{d r}-\frac{e^{\lambda}-1}{r^{2}}\right)=-8 \pi T_{1}^{1} \\
& e^{-\lambda}\left[\frac{1}{2} \frac{d^{2} v}{d r^{2}}-\frac{d \lambda}{d r} \frac{d v}{d r}+\frac{1}{4}\left(\frac{d v}{d r}\right)^{2}-\frac{1}{2 r}\left(\frac{d v}{d r}-\frac{d \lambda}{d r}\right)\right]=-\pi T_{2}^{2} \\
& T_{3}^{3}=T_{2}^{2} \\
& e^{-\lambda}\left(\frac{1}{r} \frac{d \lambda}{d r}+\frac{e^{\lambda}-1}{r^{2}}\right)=8 \pi T_{4}^{4}
\end{aligned}
$$

\section{\$3. The Solutions.}

We ussume that for a perfect fluid the material energy-tensor is such that

$$
\begin{aligned}
& T_{\mu_{z}}=0,(p \neq q) \\
& T_{1}^{\prime}=T_{2}^{2}=T_{\mathrm{z}}{ }^{3}=-p .
\end{aligned}
$$

where $p$ is the hydrostatic pressure, and

$$
T_{\star}+=\rho,
$$

the density of the fluid referred to the coordinate system $(r, \theta, \phi, t)$.

Hence, equating $T_{1}^{1}=T_{2}^{\prime 2}$, we obtain from (5) and (6)

$$
\frac{1}{2} \frac{d^{2} v}{d r^{2}}-\frac{3 \lambda}{d r} \frac{d v}{a r}+\frac{3}{4}\left(\frac{d \nu}{d r}\right)^{2}-\frac{1}{2 r} \frac{d v}{d r}-\frac{1}{2 r} \frac{d \lambda}{d r}+\frac{e^{\lambda}-1}{r^{2}}=0
$$

The equation (9) will be satisfied if the following two equations are satistied :

$$
\begin{aligned}
& \frac{1}{2} \frac{d^{2} v}{d r^{2}}+\frac{1}{4}\left(\frac{d v}{d r}\right)^{2}-\frac{1}{2 r} \frac{d v}{d r}=0, \ldots \ldots \\
& \frac{1}{2 r} \frac{d \lambda}{d r}+\frac{d \lambda}{4} \frac{d \lambda}{d r} \frac{d v}{d r}-\frac{1}{r^{2}}\left(e^{\lambda}-1\right)=0
\end{aligned}
$$

Equation (10) can be written

$$
2 \frac{d^{2} v}{d r^{2}}+\left(\frac{d v}{d r}\right)^{2}-\frac{2}{r} \frac{d v}{d r}=0
$$

Multiplying by $e^{\frac{1}{2}}$, and putting $z=e^{\frac{1}{r}}$, we have

$$
\frac{d^{2} z}{d r^{2}}+\frac{1}{r} \frac{d z}{d r}=0
$$


whence

$$
z=\beta r^{2}+\delta,
$$

$\beta, \delta$ being arbitrary constants, and

$$
e^{y}=\left(\beta r^{2}+\delta\right)^{2}
$$

Equation (11) can be written

$$
\frac{e^{\lambda}-1}{r^{2}}=\frac{1}{2} \frac{d \lambda}{d r} \cdot f
$$

where

$$
f=\frac{1}{r}+\frac{1}{2} \frac{d v}{d r}=\frac{3 \beta r^{2}+\delta}{r\left(\beta r^{2}+\delta\right)} .
$$

Putting

$$
\begin{aligned}
1-e^{\lambda} & =z \text {, we get } \\
\frac{d z}{z} & =\frac{2\left(\beta r^{2}+\delta\right) d r}{r\left(3 \beta r^{2}+\delta\right)},
\end{aligned}
$$

whence

$$
1-e^{-\lambda}=z=\frac{c r^{2}}{\left(3 \beta r^{2}+\delta\right)^{2 / 3}},
$$

$c$ being an arbitrary constant, so that

$$
e^{\lambda}=\frac{\left(3 \beta r^{2}+\delta\right)^{2 / 3}}{\left(3 \beta r^{2}+\delta\right)^{2 / 3}-c r^{2}}
$$

Thus we find that the field (1) with the values of $\lambda$ and $\nu$ given by (12) and (13) satisfies the gravitational equations.

From (8) we obtain

$$
\begin{aligned}
8 \pi T_{\downarrow} & =\frac{e^{-\lambda}}{r} \frac{d \lambda}{d r}+\frac{1-e^{-\lambda}}{r^{2}}=\frac{z}{r^{2}}\left(1+\frac{2}{r f^{\prime}}\right) \\
& =\frac{c\left(5 \beta r^{2}+3 \delta\right)}{\left(3 \beta r^{2}+\delta\right)^{5 / 3}},
\end{aligned}
$$

or,

$$
\rho=T_{4}^{4}=\frac{c}{8 \pi} \frac{5 \beta r^{2}+3 \delta}{\left(3 \beta r^{2}+\delta\right)^{5 / 3}}
$$

From (5) we get

$$
\begin{aligned}
p & =-T_{1}^{1}=-T_{2}^{\prime 2}=-T_{3}^{3} \\
& =\frac{1}{8 \pi\left(\beta r^{2}+\delta\right)\left(3 \beta r^{2}+\delta\right)^{2 / 3}}\left[4 \beta\left\{\left(3 \beta r^{2}+\delta\right)^{2 / 3}-c r^{2}\right\}-c\left(\beta r^{2}+\delta\right)\right](
\end{aligned}
$$


There are three arbitrary constants in these solutions, viz., $\beta, \delta$ and $c$. We make $\delta=1$. The other two constants $\beta$ and $c$ are then determined from the conditions:

(1) the pressurc $p=0$ at $r=a$, the radius of the sphere,

(2) the density at the centre $=\rho_{0}$, a given quantity.

We thus obtain

$$
c=\frac{4 \beta\left(3 \beta a^{2}+1\right)^{2 / 3}}{5 \beta a^{2}+1}=\frac{8 \pi}{3} \rho_{0}
$$

and

$$
\rho=\frac{5 \beta r^{2}+3}{3\left(3 \beta r^{2}+1\right)^{5 / 3}} \cdot \rho
$$

We therefore nrive at the following result:

The gravitational field of a heterogeneous fluid sphere uhose density at the centre is $\rho_{0}$ and the density at a distance $r$ from the centre is given by $\rho=\frac{5 \beta r^{2}+3}{3\left(\beta r^{2}+1\right)^{3 / 3}} \rho_{0}$, has the metric

$$
d s^{2}=-e^{2} d r^{2}-r^{2} d \theta^{2}-r^{2} \sin ^{2} \theta d \phi^{2}+e^{2} d t^{2},
$$

where $e^{\lambda}=\frac{\left(3 \beta r^{2}+1\right)^{2 / 3}}{\left(3 \beta r^{2}+1\right)^{2 / 3}-\frac{8 \pi \rho_{0}}{3} r^{2}}$, and $\epsilon^{\nu}=\left(\beta r^{2}+1\right)^{\circ}$,

where $\beta$ is a constant expressible in terms of $\rho_{0}$ by means of (16).

\section{$\$ 4$. The general problem.}

The general problem of a fluid sphere with an arbitrary law of density may be treated in the following manner.

Let $\rho=\psi(r)$ be the density of any point distant $r$ from the centre. From equation (8), we obtain, * on integration

whence

$$
\left(1-e^{-\lambda}\right) r=A+8 \pi \int \psi\langle r\rangle r^{2} d r
$$

$$
\begin{aligned}
& e^{\lambda}=\left[1-\frac{1}{r}\left\{A+8 \pi \int \psi(r) r^{2} d r\right\}\right]^{-1} \\
& =\phi(r), \text { say. }
\end{aligned}
$$

* Gf. Combrider, Phil. Mag., Jau. 1926. 
From the condition $T_{1}^{1}=T_{2}{ }^{2}=T_{3}^{3}$, that is, from equation (9), we have

$$
2 \frac{d^{2} v}{d r^{2}}+\left(\frac{d v}{d r}\right)^{2}-\left(\frac{2}{r}+\frac{d \lambda}{d r}\right) \frac{d v}{d r}-\frac{2}{r} \frac{d \lambda}{d r}+\frac{4\left(e^{\lambda}-1\right)}{r^{2}}=0,
$$

or,

$e^{d v}\left\{2 \frac{d^{2} v}{d r^{2}}+\left(\frac{d v}{d r}\right)^{2}\right\}-e^{d v} \frac{d v}{d r}\left(\frac{2}{r}+\frac{d \lambda}{d r}\right)-e^{\frac{1}{2} v}\left\{\frac{2}{r} \frac{d \lambda}{d r}+\frac{4\left(e^{\lambda}-1\right)}{r^{2}}\right\}=0$,

or, putting $e^{\frac{1}{2} v}=z$,

$$
4 \frac{d^{2} z}{d r^{2}}-2\left(\frac{2}{r}+\frac{d \lambda}{d r}\right) \frac{d z}{d r}-\left\{\frac{2}{r} \frac{d \lambda}{d r}+\frac{4\left(e^{\lambda}-1\right)}{r^{2}}\right\} z=0,
$$

or,

$$
\frac{d^{2} z}{d r^{2}}+v_{1} \frac{d z}{d r}+v_{2} z=0
$$

where

$$
\begin{aligned}
& v_{1}=-\frac{1}{2}\left(\frac{2}{r}+\frac{d \lambda}{d r}\right) \\
& v_{2}=-\frac{1}{4}\left\{\frac{2}{r} \frac{d \lambda}{d r}+\frac{4\left(e^{\lambda}-1\right)}{r^{2}}\right\}
\end{aligned}
$$

Hence we obtain the theorem :

The gravitational field of a heterogeneous fluid sphere, whose density at any point distant $r$ from the centre is $\psi(r)$, is given by the metric (1), in which

$$
\begin{aligned}
e^{\lambda} & =\phi(r) \\
& =\left[1-\frac{1}{r}\left\{A+8 \pi \int \psi(r) r^{2} d r\right\}\right]^{-1}
\end{aligned}
$$

and $e^{\nu}$ is obtained from the equation

$$
\frac{d^{2} z}{d r^{2}}+v_{1} \frac{d z}{d r}+v_{2} z=0
$$

where

$$
\begin{aligned}
z & =e^{1 / 2 v} \\
v_{1} & =-\left(\frac{1}{r}+\frac{1}{2 \phi} \frac{d \phi}{d r}\right) \\
\eta_{2} & =-\left\{\frac{1}{2 r \phi} \frac{d \phi}{d r}+\frac{1}{r^{2}}(\phi-1)\right\} .
\end{aligned}
$$


The theorem may be made use of to discover laws of density for which the field is exactly determinable.

\section{\$5. Remarks.}

It is seen from (16) that, when the constant $\beta=0$ we have also $c=0$, and then from (14), $\rho=0$; so that the corresponding space is empty. The corresponding values of $e^{\lambda}$ and $e^{\nu}$ are each unity from (12) and (13), and the space degenerates, as might be expected, into a galilean space time of special relativity.

From (17), we see that

$$
\rho=\frac{5 \rho_{0}}{3^{8 / 3} \cdot \rho^{2 / 3}} \cdot \frac{1}{r^{4 / 3}}\left(1+\frac{2}{45 \beta r^{2}}+\ldots\right),
$$

which shews that $\rho$ is of order $r^{-4}$, so that the density diminishes but gradually as the distance from the centre increases.

If, instead of (4), the modified equations of gravitation are used in the form (with no summation convention)

we get

$$
K_{p p}-\frac{1}{2} g_{p p} K+\alpha g_{p l}=-8 \pi{ }_{p p},(p=1,9,3,4) \ldots \ldots(20)
$$

or,

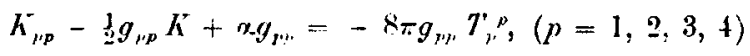

$$
K_{p p}-\frac{1}{2} g_{p p} K=-8 \pi g_{m}\left(T_{p}{ }^{\prime}+\frac{\alpha}{8 \pi}\right),(p=1,2,3,4) \ldots
$$

These differ from the equations (5) ... (8), only in the fact that $T_{p}{ }^{p}$ is increased by the quantity $\frac{\alpha}{8 \pi}$,

If, in this case, $T_{p}{ }^{p}=0$, i.e. if the space is empty, the corresponding solution is known to be the space-time of de Sitter's world.

I wish to express my sense of appreciation of the kind interest Professor Whittaker takes in my work. 